\title{
QTLs for Resistance to Fusarium Ear Rot in a Multiparent Advanced Generation Intercross (MAGIC) Maize Population
}

\author{
A. Butrón, ${ }^{1, \dagger}$ R. Santiago, ${ }^{2,3}$ A. Cao, ${ }^{1}$ L. F. Samayoa, ${ }^{4}$ and R. A. Malvar ${ }^{1}$ \\ ${ }^{1}$ Misión Biológica de Galicia (CSIC), Box 28, Pontevedra 36080, Spain; ${ }^{2}$ Facultad de Biología, Departamento de Biología Veg- \\ etal y Ciencias del Suelo, Universidad de Vigo, As Lagoas Marcosende, Vigo 36310, Spain; ${ }^{3}$ Agrobiología Ambiental, Calidad de \\ Suelos y Plantas (UVIGO), Unidad Asociada a la MBG (CSIC), Pontevedra 36143, Spain; and ${ }^{4}$ Department of Crop \& Soil Sci- \\ ences, North Carolina State University, Raleigh, NC 27695, U.S.A.
}

\begin{abstract}
Alternative approaches to linkage and association mapping using inbred panels may allow further insights into loci involved in resistance to Fusarium ear rot and lead to the discovery of suitable markers for breeding programs. Here, the suitability of a maize multiparent advancedgeneration intercross population for detecting quantitative trait loci (QTLs) associated with Fusarium ear rot resistance was evaluated and found to be valuable in uncovering genomic regions containing resistance-associated loci in temperate materials. In total, 13 putative

minor QTLs were located over all of the chromosomes, except chromosome 5 , and frequencies of favorable alleles for resistance to Fusarium ear rot were, in general, high. These findings corroborated the quantitative characteristic of resistance to Fusarium ear rot in which many loci have small additive effects. Present and previous results indicate that crucial regions such as 210 to $220 \mathrm{Mb}$ in chromosome 3 and 166 to $173 \mathrm{Mb}$ in chromosome 7 (B73-RefGen-v2) contain QTLs for Fusarium ear rot resistance and fumonisin content.
\end{abstract}

Plant breeding has emerged as an effective and environmentally safe method to control fungal infections and reduce mycotoxin levels in susceptible crops (Eller et al. 2008; Jouany 2007). Because there is a high level of genetic variability for resistance to fumonisin accumulation and this trait shows moderate to high heritability, developing maize varieties with greater resistance levels to kernel fumonisin accumulation through breeding is probable (Bolduan et al. 2009; Löffler et al. 2010, 2011; Robertson et al. 2006; Santiago et al. 2013). However, direct selection for mycotoxin contamination is expensive and time consuming; therefore, indirect selection criteria that are cheaper and less time consuming are necessary (Eller et al. 2010). Fusarium ear rot has been proposed as a suitable target trait for indirect selection based on the previously reported high-level genotypic correlations between Fusarium ear rot and fumonisin content (Bolduan et al. 2009; Eller et al. 2010; Löffler et al. 2010, 2011; Presello et al. 2011).

Several approaches have been used to detect loci involved in maize resistance to Fusarium ear rot. Biparental mapping populations developed from crosses between inbred lines with contrasting resistance levels to Fusarium ear rot have been widely used (AbdelRahman et al. 2016; Chen et al. 2012, 2016; Ding et al. 2008; Giomi et al. 2016; Ju et al. 2017; Li et al. 2011; Maschietto et al. 2017; Pérez-Brito et al. 2001; Robertson-Hoyt et al. 2006). In these studies,

${ }^{\dagger}$ Corresponding author: A. Butrón; E-Mail: abutron@mbg.csic.es

Funding: This research was funded by the Plan Estatal de Ciencia y Tecnologia de España and European Union funds under the FEDER program within the projects AGL2015-67313-C2-1-R and AGL2015-67313-C2-2-R, and the Autonomous Government of Galicia, Spain, project IN607A/013. This research has been developed in part at the "Agri-Food Research and Transfer Centre of the Water Campus (CITACA) at the University of Vigo (Spain)", which is economically supported by the Galician Government. R. Santiago acknowledges postdoctoral contract "Ramón y Cajal" financed by the Ministry of Economy and Competiveness (Spain), Vigo University, and the European Social Fund.

The author(s) declare no conflict of interest.

Accepted for publication 24 October 2018.

(c) 2019 The American Phytopathological Society quantitative trait loci (QTLs) for resistance to Fusarium ear rot were detected in all chromosomes; however, the overlapping of QTLs across studies was rare. The percentage of total variability explained was low, and each QTL accounted for only a small percentage of phenotypic variability. Because no major QTLs acting across different genetic backgrounds were found, marker-assisted selection for resistance to Fusarium ear rot using these markers was not encouraged (Lanubile et al. 2017). Later, association mapping studies exploring variability for resistance to Fusarium ear rot in diverse inbred panels confirmed the relatively small effects of many underlying causal variations and revealed the low frequencies of many variants (Chen et al. 2016; Ju et al. 2017; Zila et al. 2013, 2014). Although the mapping resolution was greatly improved in association studies compared with QTL mapping in biparental populations, the former were not powerful enough to uncover the genetics of maize resistance to Fusarium ear rot. Low frequencies of minor alleles and small gene effects for Fusarium ear rot among panels of inbred samples hampered the detection of significant associations between single-nucleotide polymorphism (SNP) loci and variation for maize resistance to Fusarium ear rot (Zila et al. 2013, 2014).

In this context, mapping approaches with intermediate characteristics between biparental and association mapping populations could be more useful in discovering loci involved in resistance to Fusarium ear rot and suitable associated markers for breeding programs. Multiparent advanced-generation intercross (MAGIC) populations provide the capability to capture greater levels of genetic diversity and increased mapping resolutions compared with those of biparental populations because alleles from diverse founder lines are segregating and the intercrossing of founders for several generations before selfing leads to an amplified number of recombination events (Cavanagh et al. 2008; Huang et al. 2015; Verbyla et al. 2014). Because the number of founders is limited and all founders have an equal probability of contributing to the genetic makeup of a line, frequencies of rare alleles are greater in MAGIC populations compared with in association panels, and the power for QTL detection is increased (Holland 2015). However, because MAGIC populations also have some disadvantages compared with association panels and biparental populations, such as lower levels of genetic diversity and lower frequencies of rare alleles, respectively, results from different mapping populations may be complementary.

To generate an efficient MAGIC population several factors must be taken into account: (i) founder selection to achieve an optimal 
level of genetic and phenotypic diversity; (ii) design and selection of funnels to create an outbred population that is robust enough to account for maternal effects, population structure, and segregation distortion; and (iii) advanced generations of intercrossing to increase the recombination rate in the population (Huang et al. 2015). MAGIC populations have been developed in many crops such as barley (Sannemann et al. 2015), wheat (Huang et al. 2012), rice (Meng et al. 2016a,b), maize (Dell'Acqua et al. 2015), tomato (Pascual et al. 2015), and cotton (Islam et al. 2016). In general, the average linkage disequilibrium in those populations was below 0.2 within physical distances of approximately 0.5 to $2.0 \mathrm{Mbp}$, while confidence intervals for QTLs located in biparental populations often span more than $10 \mathrm{Mbp}$. In addition, the resolution of MAGIC populations could be improved if successive intercrossing was performed before selfing. Consequently, the average support intervals of QTLs detected in a MAGIC tomato population were $30 \%$ smaller than those located using a biparental population and, in a panel of 163 accessions, the number of QTLs detected in the MAGIC population was twice the number obtained using association mapping (Pascual et al. 2016). In the current study, the suitability of a maize MAGIC population for detecting QTLs for Fusarium ear rot was evaluated. In addition, prospects for using this MAGIC population as a base

\section{Eight Founders}

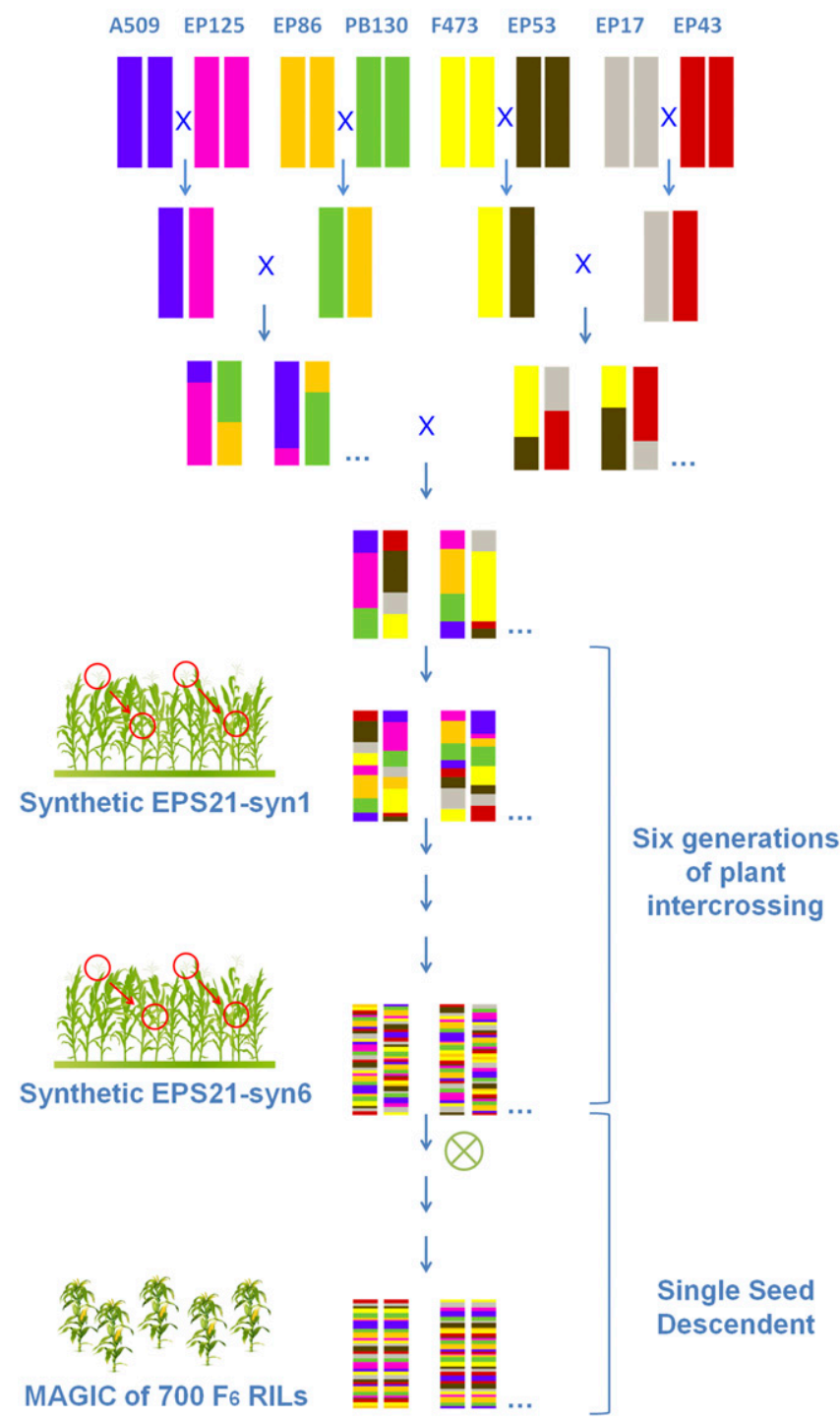

Fig. 1. Scheme of the procedure used to develop the recombinant inbred lines (RIL) that constitute the multiparent advanced-generation intercross (MAGIC) population derived from intercrossing eight inbred founders. material in marker-assisted selection for resistance to kernel fumonisin accumulation were assessed.

\section{Materials and Methods}

A maize synthetic variety, EPS21, constructed from eight inbred lines with partial resistance to the Mediterranean corn borer (Butrón et al. 2003, 2009) was used to build the MAGIC population. The inbred lines A509, EP17, EP43, EP53, EP125 (named CO125 in previous studies because this inbred line was deposited in the Misión Biológica de Galicia germplasm bank in 1985 from the Instituto de Biología de Barcelona as a version of the Canadian inbred line CO125; however, recent molecular studies have indicated that the two lines are clearly different [unpublished work]), F473, EP86, and PB130 belong to different heterotic groups (the Reid group was not represented) and showed contrasting resistance levels to Fusarium ear rot in a previous evaluation (Santiago et al. 2013). EP17, EP86, EP125, and A509 are partially resistant to Fusarium ear rot, while EP43, EP53, and F473 are intermediate. PB130 is highly susceptible to Fusarium ear rot. Once the synthetic EPS21 variety was constructed, six generations of recombination were performed before selfing was initiated (Fig. 1). In each generation of recombination, 300 EPS2 1 seeds were sown in 10 rows each, with 15 hills and 2 seeds per hill. The seedlings were later thinned to one plant per hill, leaving 150 plants for plant-to-plant crosses. Each plant was used only once as male or female, resulting in at least 50 ears. Equal numbers of seed were bulked from each ear, and another generation of recombination was carried out. Finally, approximately 700 recombinant inbred lines (RILs) were obtained by successively selfing generations having a single-seed descent.

In total, 672 RILs were evaluated, along with the eight inbred founders, in 2014 at Pontevedra, Spain $\left(42^{\circ} 24^{\prime} \mathrm{N}, 8^{\circ} 38^{\prime} \mathrm{W}, 20 \mathrm{~m}\right.$ above sea level), following an augmented design with 16 blocks. In each block, 42 nonreplicated RILs were included, along with the eight inbred founders of the MAGIC population. Plots consisted of single rows of 13 plants. In addition, 695 RILs and seven of the founders (no seed of PB130 was available) were evaluated in 2016 at Pontevedra following a 26-by-27 $\alpha$ design with two replicates. Plots consisted of single rows of 19 plants. In both years, 7 to 14 days after silking (calculated as the day in which $50 \%$ of plants in the row were shedding silks), five primary ears per plot were inoculated with $2 \mathrm{ml}$ of a spore suspension of an aggressive toxigenic isolate of Fusarium verticillioides (Cao et al. 2014). The spore suspension contained $10^{6}$ spores $/ \mathrm{ml}$ and was injected into the center of the ear using a fourneedle vaccinator that perforated the husks and injured three to four kernels (Reid et al. 1996). Inoculated ears from each row were collected 2 months after inoculation and were individually rated for Fusarium ear rot using a seven-point scale $(1=$ no visible disease symptoms and $2=1$ to $3,3=4$ to $10,4=11$ to $25,5=26$ to 50 , $6=51$ to 75 , and $7=76$ to $100 \%$ of kernels exhibiting visual symptoms of infection) devised by Reid and Zhu (2005). A combined analysis of variance was performed for Fusarium ear rot using the procedure PROC MIXED of SAS, in which replicate and year were random factors and RIL was fixed (SAS Institute 2008). Then, the best linear unbiased estimator for each RIL in each year was estimated using the SAS mixed-model procedure (PROC MIXED), in which RIL was a fixed effect and block was a random effect (replicate in 2016 was also considered as a random effect). Owing to germination failure, the best linear unbiased estimator data in both years were only available for 352 RILs, and subsequent statistical analyses were performed with that subset of the original MAGIC population. The average RIL data across years were used to perform genomewide association studies (GWAS). The Kruskal-Wallis nonparametric test was performed with the procedure Proc NPAR1WAY of SAS to determine differences in Fusarium ear rot among inbred founders.

The 352 RILs and the eight inbred founders were genotyped in the Biotechnological Institute of the Cornell University for approximately 1,000,000 SNPs by a methodology of genotyping based on the sequence. The RILs with an excess of heterozygotes were removed, rendering a final set of 339 RILs. In the MAGIC population, monomeric and multiallelic SNPs and insertion and deletion polymorphisms were excluded. Then, the data set was filtered to exclude 
SNPs with more than $20 \%$ missing genotype data and minor allele frequency less than 0.05. In total, 58,556 filtered SNPs distributed across the maize genome were used for GWAS analysis.

Heritabilities $\left(\hat{h}^{2}\right)$ across environments were estimated for Fusarium ear rot on a family-mean basis, as described by Holland et al. (2003). The genetypic $\left(r_{g}\right)$ and phenotypic $\left(r_{p}\right)$ correlations between Fusarium ear rot and days to shedding pollen and silks were computed following Holland (2006). These statistical analyses, along with those for computing estimations of genetic variance for Fusarium ear rot, were performed using the SAS statistical package (SAS Institute 2008). GWAS analysis based on mixed linear models was performed in FarmCPU (Liu et al. 2016). The recommended $P$ value threshold was calculated as the $90 \%$ quantile value of the vector containing $P$ values from 1,000 run permutations ( $P$ value threshold $=2.59 \mathrm{E}-06)$. SNPs at the upper right section of the quantile-quantile (Q-Q) plot of the model that lie outside the $95 \%$ confidence interval for the null hypothesis of no association between the SNP and the trait were also presented (Gowda et al. 2015). Linkage disequilibrium in genomic regions containing significant SNPs was visually explored using TASSEL (Bradbury et al. 2007).

\section{Results}

There were differences among founders of the maize MAGIC population for Fusarium ear rot (Kruskal-Wallis statistic $=44.55,7$ degrees of freedom, $P$ value $<0.0001)$. Thus, the genetic variance for Fusarium ear rot in the MAGIC population was significantly different from zero $(0.43 \pm 0.06)$, and the heritability was moderate $(0.56 \pm$ $0.04)$. The genetic variance for days to shedding silks $(9.57 \pm 1.00)$ was also significantly different from zero but the genotypic correlation coefficient between Fusarium ear rot and days to shedding silks $(-0.03 \pm 0.09)$ was not significant. In addition, the combined analysis of variance showed significant differences among RILs for Fusarium ear $\operatorname{rot}(F=2.35, P<0.0001)$ but the year-RIL interaction was not significant $(Z=1.16, P=0.1227)$.
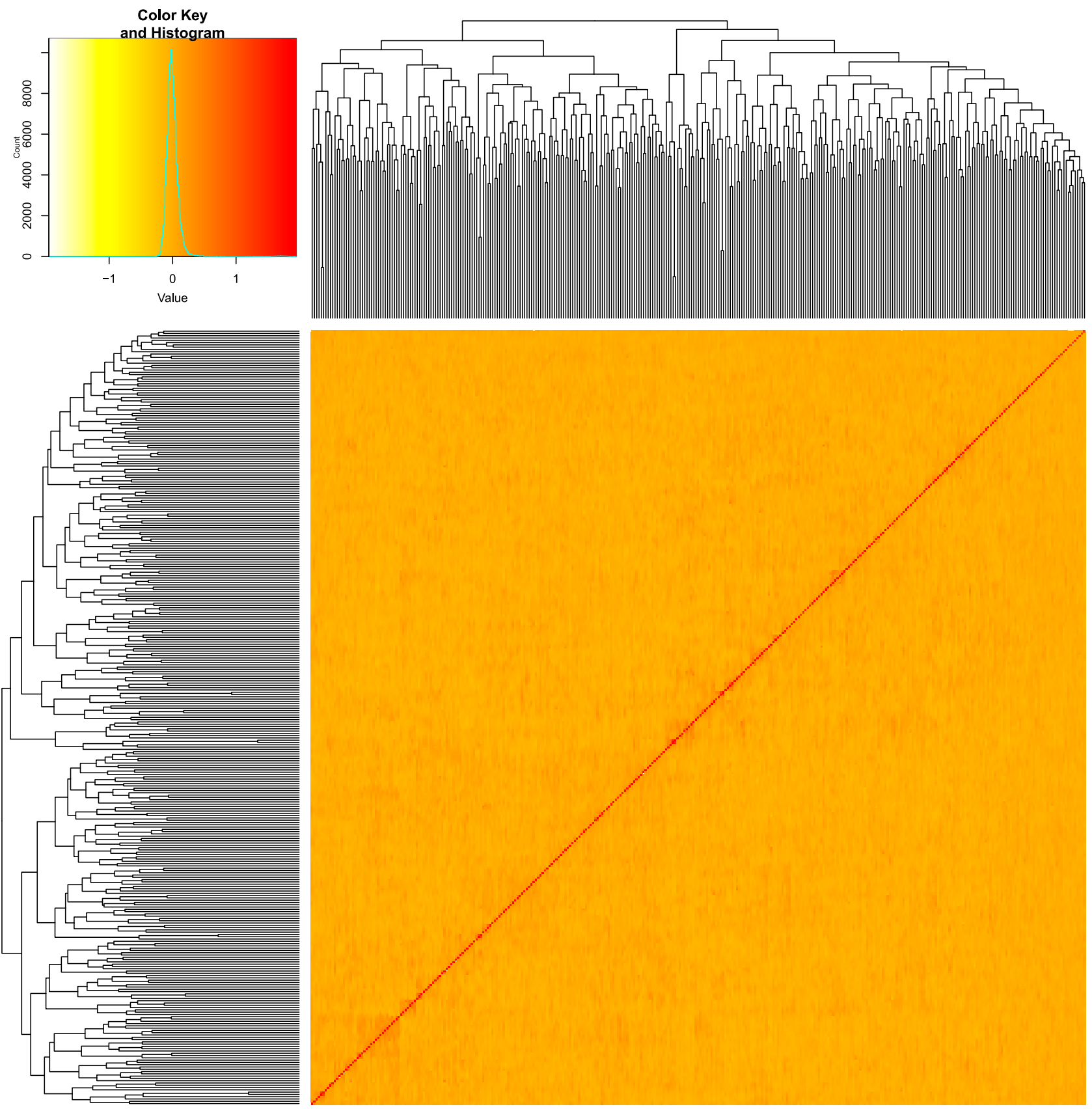

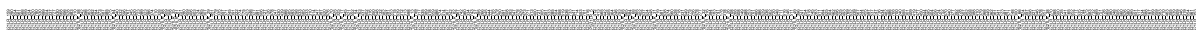

Fig. 2. Heat map of the values of the Kinship matrix used in the mixed linear model for Fusarium ear rot. 
The Van Raden Kinship used for the GWAS analysis is shown in Figure 2 and indicates that the genetic relatedness among RILs is not even across the MAGIC population. Assortative mating during synthetic development could explain the structure observed in the MAGIC population because mating between individuals with similar earliness traits could unintentionally occur.

In total, 13 SNPs were located in the upper part of the Q-Q plot (Fig. 3; $P$ values $<0.0001$ ), outside of the $95 \%$ confidence interval for no significant association between SNP and Fusarium ear rot, and these SNPs could be considered as linked to putative QTLs. However, only three presented $P$ values below the threshold calculated by 1,000 run permutations and only one was under the 0.05 Benjamini-Hochberg cutoff (Fig. 4). For most significant SNPs, frequencies of favorable alleles for resistance to Fusarium ear rot were greater than those of unfavorable alleles, and even founders that were highly susceptible to Fusarium ear rot such as PB130 seemed to carry

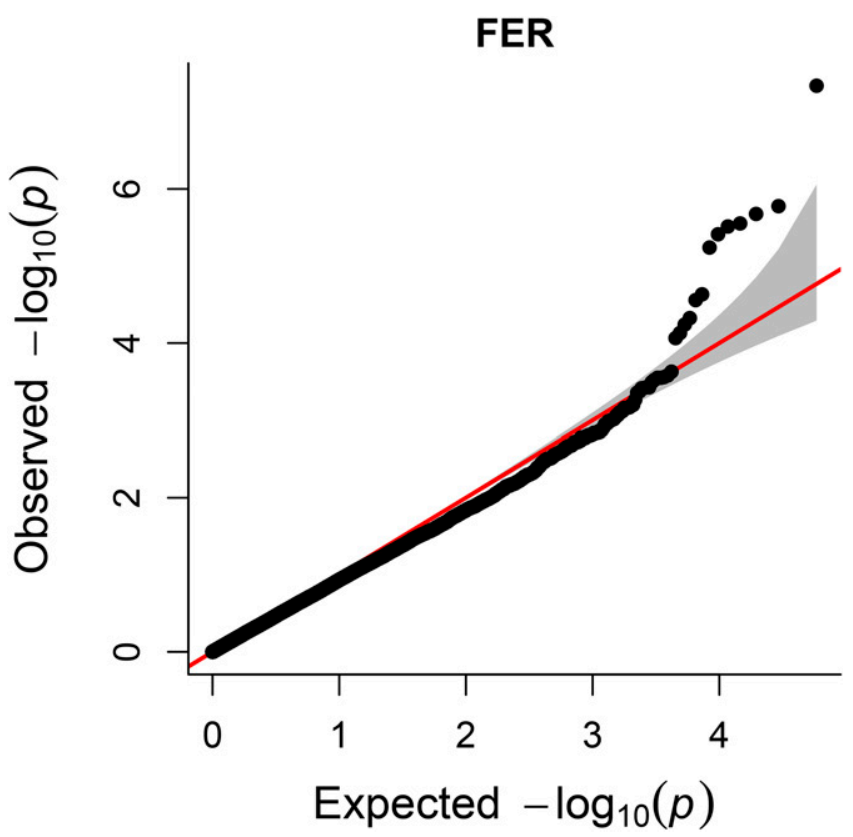

Fig. 3. Quantile-quantile plots of a mixed linear model for Fusarium ear rot in a maize multiparent advanced-generation intercross population. alleles for some significant resistance-associated SNPs. The most resistant founder, A509, could be the result of many gene actions.

The approximate supporting interval for each QTL was established by visually checking the linkage disequilibrium (LD) heatmap plot of the genomic region that contains the SNPs. The supporting interval was considered the region in which appreciable LD was observed between SNPs $\left(r^{2}>0.3\right)$. Supporting intervals for QTLs were, in general, less than $10 \mathrm{Mbp}$, except for QTLs located in centromeres or near the centromeres, where LD is exceptionally high (Table 1). LD was also explored across the entire genome, and the average minimum distance between SNPs to achieve an LD value of $r^{2}<0.30$ was more than $1 \mathrm{Mbp}$ (Fig. 5), which indicated that the marker density used here (Fig. 6) was more than sufficient to discern significant associations between SNPs and Fusarium ear rot.

\section{Discussion}

The experimental conditions such as the design and inoculation technique used were sufficient to produce a phenotypic value for each particular RIL for Fusarium ear rot that was highly stable across environment, as indicated by the year-environment interaction for Fusarium ear rot being not significant. There was significant variability in the MAGIC population for Fusarium ear rot, suggesting that these RILs could be used to locate QTLs for resistance to Fusarium ear rot. In addition, the heritability estimate for Fusarium ear rot was moderate but slightly lower than estimates obtained in panels of diverse inbred lines (Chen et al. 2016; Ju et al. 2017; Zila et al. 2013, 2014). The reported characteristics of the MAGIC population such as moderate heritability for Fusarium ear rot and low structure, along with moderate frequencies of favorable allelic variants, indicated that this population could be a good complement to diverse inbred panels in the quest to uncover genomic regions implicated in Fusarium ear rot resistance among temperate materials because frequencies of favorable alleles for resistance to Fusarium ear rot are low in inbred panels, especially in temperate inbred groups (Zila et al. 2013, 2014). In addition, because the inbred founders also differed in fumonisin accumulation in kernels, identifying promising QTLs for Fusarium ear rot resistance will encourage the search for QTLs associated with fumonisin accumulation in the future (Santiago et al. 2013).

Another advantage of the current MAGIC population is that Fusarium ear rot did not appear to be correlated with days to silking. Therefore, no unfavorable effects on maturity would be expected when these favorable alleles for resistance to Fusarium ear rot are stacked in new breeding materials or transferred into elite inbred lines. However, plant maturity has been associated with Fusarium ear rot in

\section{FER}

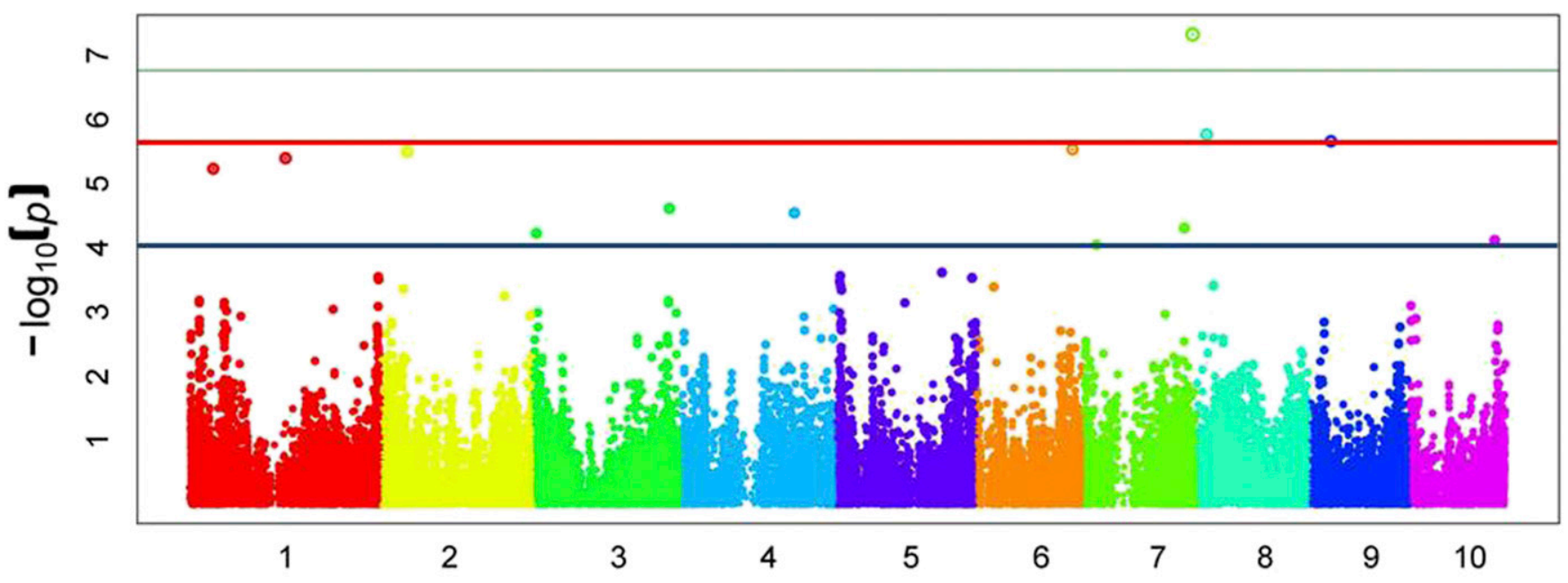

Fig. 4. Manhattan plot of a mixed linear model for Fusarium ear rot (FER) in a maize multiparent advanced-generation intercross population of recombinant inbred lines. Singlenucleotide polymorphisms (SNPs) above the green horizontal line surpassed the 0.05 Benjamini-Hochberg cutoff, above the red horizontal line the $P$ value threshold obtained by 1,000 run permutations (2.59E-06), and above the blue horizontal line the $P$ value $=0.0001$ (samples with lower $P$ values were located outside of the $95 \%$ confidence interval for no significant association between SNP and FER). 
biparental populations in which large LD blocks are present (Maschietto et al. 2015) and in panels of diverse inbred lines in which a high percentage of the phenotypic variance for days to shedding silks was explained by the background genetic variance (Chen et al. 2016; Zila et al. 2013). The reduced linkage blocks of the MAGIC population compared with biparental populations and the expected lower effect of pedigree compared with panels of diverse inbred lines could be beneficial to eliminate the linkage drag of maturity on Fusarium ear rot.

In total, 13 putative QTLs were detected but only 3 had $P$ values above the recommended threshold based on permutations. This was not unexpected because many loci with small effects appear to be involved in resistance to Fusarium ear rot, making the detection of highly reliant QTLs difficult (Santiago et al. 2015). In addition, many favorable variants for significant SNPs seemed to be frequent among the founders of the MAGIC population, which contributed to its reduced power in QTL detection.

The mean supporting interval for each QTL (except QTLs in centromeres) was less than $10 \mathrm{Mbp}$ and was within the range of supporting intervals for QTLs found in MAGIC populations in other crops (Huang et al. 2012; Pascual et al. 2015, 2016). Those supporting intervals could be reduced using more RILs but the mapping resolutions of MAGIC populations are still insufficient to achieve the goal of identifying candidate genes for QTLs with small effects (Valdar et al. 2006). In the best scenario, the confidence interval was just under 0.6 centimorgans.

In the current MAGIC population, significant SNPs were found in bins in which QTLs had been previously detected using biparental

Table 1. Summary of quantitative trait loci (QTLs) for resistance to Fusarium ear rot found in a maize multiparent advanced-generation intercross population under inoculation with Fusarium verticillioides in 2 years

\begin{tabular}{|c|c|c|c|c|c|c|c|c|c|}
\hline QTL & SI $(\mathbf{M b})^{\mathbf{a}}$ & $\operatorname{Bin}^{\mathbf{b}}$ & SNP position ${ }^{c}$ & $P$ value & Effect & Alleled $^{d}$ & Favorable allele donors & Founders with no data & $\operatorname{Ref}^{\mathrm{e}}$ \\
\hline 1 & $32-44$ & 1.03 & $37,132,524$ & $5.73 \mathrm{E}-06$ & 0.16 & 0.30 & EP17, EP43, PB130 & $\ldots$ & 1 \\
\hline 2 & - & 1.05 & $150,112,685$ & $3.90 \mathrm{E}-06$ & 0.16 & 0.34 & EP43, EP53, F573 & $\ldots$ & $\ldots$ \\
\hline 3 & - & 2.04 & $38,985,670$ & $3.07 \mathrm{E}-06$ & -0.15 & 0.59 & EP17, EP53, PB130 & EP43, F473 & $\ldots$ \\
\hline 4 & $3-6$ & $3.01-3.02$ & $3,994,836$ & $5.77 \mathrm{E}-06$ & -0.17 & 0.85 & A509, EP125, EP43, EP53, EP86, F473 & PB130 & . \\
\hline 5 & $210-220$ & $3.08-3.09$ & $211,757,621$ & $2.34 \mathrm{E}-05$ & -0.14 & 0.77 & A509, EP125, EP43, EP53, F473, РB130 & $\ldots$ & 3 \\
\hline 6 & $170-180$ & 4.07 & $174,915,620$ & $2.78 \mathrm{E}-05$ & -0.19 & 0.84 & All except F473 & $\ldots$ & $\ldots$ \\
\hline 7 & $138-152$ & 6.05 & $151,037,139$ & $2.82 \mathrm{E}-06$ & -0.15 & 0.58 & EP17 & EP86, F473, PB130 & 5 \\
\hline 8 & - & 7.02 & $19,419,592$ & $8.66 \mathrm{E}-05$ & -0.20 & 0.91 & A509, EP125, EP17, EP43, РB130 & EP86, F473 & $\ldots$ \\
\hline 9 & $150-161$ & $7.03-7.04$ & $156,501,462$ & 4.73E-05 & -0.19 & 0.91 & A509, EP125, EP43, EP53, EP86, F473 & PB130 & 1,5 \\
\hline 10 & $166-173$ & $7.04-7.05$ & $169,937,078$ & $4.59 \mathrm{E}-08$ & -0.30 & 0.91 & All except EP86 & $\ldots$ & 3,5 \\
\hline 11 & $11-17$ & 8.02 & $14,952,980$ & $1.67 \mathrm{E}-06$ & 0.16 & 0.43 & A509, EP125, EP53 & EP86 & 1 \\
\hline 12 & - & 9.03 & $33,235,644$ & $2.12 \mathrm{E}-06$ & -0.15 & 0.60 & A509, EP43, EP53, PB130 & EP17 & 2,3 \\
\hline 13 & $127-138$ & 10.05 & $132,620,492$ & $7.41 \mathrm{E}-05$ & -0.22 & 0.93 & A509, EP17, EP125, EP43, EP53, F473 & PB130 & $\ldots$ \\
\hline
\end{tabular}

${ }^{a}$ Supporting interval (SI) of the QTL, region in which appreciable linkage disequilibrium (LD) is observed between single-nucleotide polymorphisms (SNPs) $\left(r^{2}>0.3\right)$. SI could not be visualized using LD plots from TASSEL when SNPs were localized in centromeric or pericentromeric regions where LD is extremely high.

${ }^{\mathrm{b}}$ Bin in which QTLs and SNPs are located.

${ }^{c}$ Position in base pairs of the significant SNP, $P$ value for the association between polymorphic variation at the SNP and phenotypic variation for Fusarium ear rot, and difference between the average phenotypic value of the homozygotes for the more and less frequent alleles.

${ }^{\mathrm{d}}$ Proportion of the favorable allele.

${ }^{\mathrm{e}}$ References in which SNPs significantly associated with Fusarium ear rot or fumonisin content were found within the SI region: $1=\mathrm{Ju}$ et al. (2017), $2=\mathrm{Chen}$ et al (2016), 3 = Samayoa et al. (2018), 4 = Zila et al. (2014), and 5 = Coan et al. (2018).

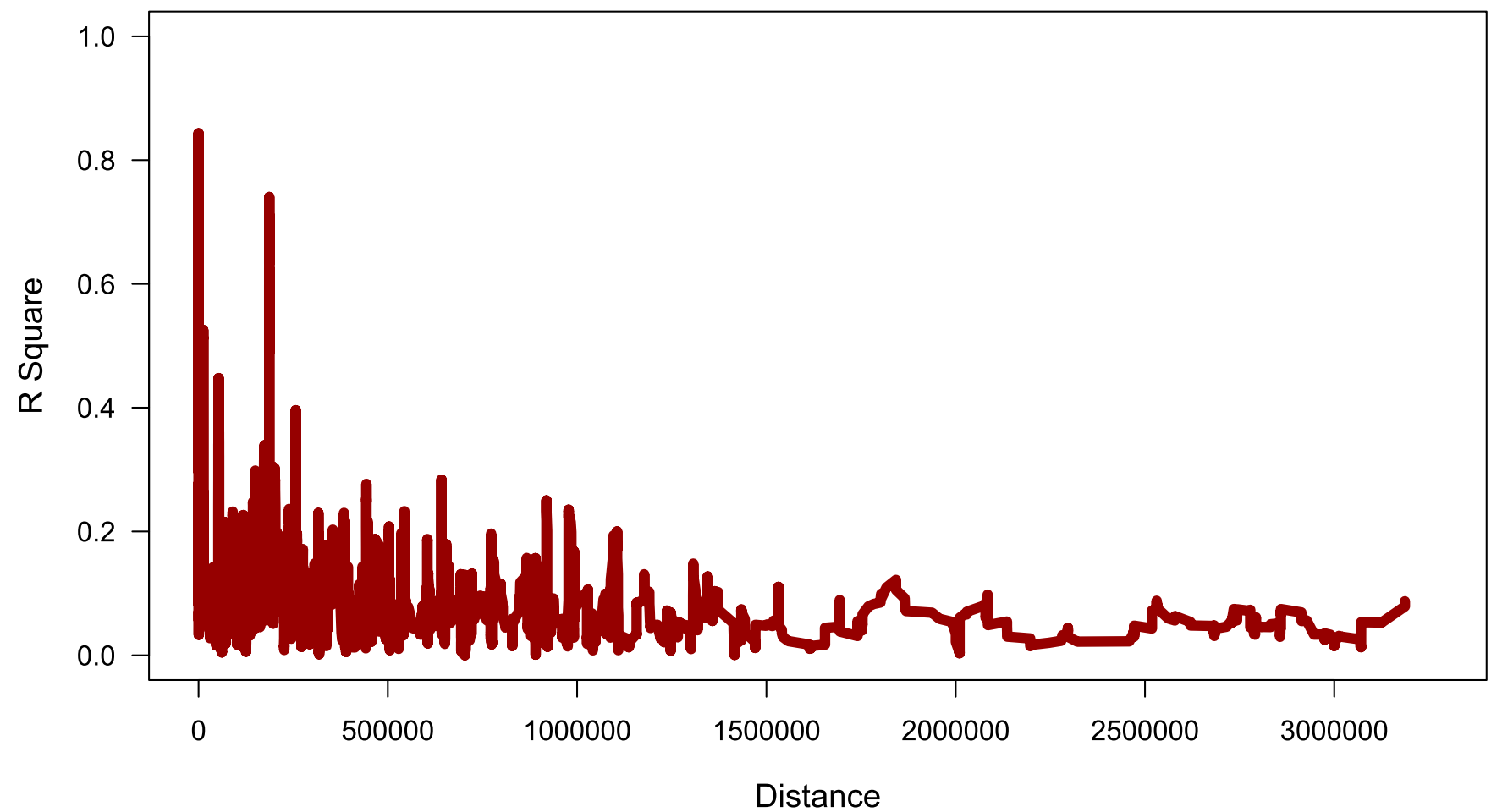

Fig. 5. Linkage disequilibrium (LD) decay over distance for pairwise markers. LD values were calculated on sliding windows with 100 adjacent single-nucleotide polymorphisms (SNPs). Each dot represents the distance between two SNPs on the window and their squared correlation coefficient. The line is the moving average of 10 adjacent markers. 
populations (Chen et al. 2012, 2016; Ding et al. 2008; Giomi et al. 2016; Ju et al. 2017; Li et al. 2011; Maschietto et al. 2017; PérezBrito et al. 2001; Robertson-Hoyt et al. 2006). The genetic variability for Fusarium ear rot at some specific regions may be underrepresented in the MAGIC population, especially in chromosome 5, in which no significant SNPs were found, because QTLs for Fusarium ear rot in chromosome 5 had already been reported. The lack of significant SNPs in regions in which significant QTLs were found in biparental populations could be the consequence of the reduced variability in the MAGIC population compared with the total variability across biparental populations, but it could also be the consequence of lower allele frequencies in the MAGIC population coupled with small additive effects that reduce the power of QTL detection compared with that in biparental populations. To explore the wider genetic variability for Fusarium ear rot among temperate materials, a MAGIC population with more inbred founders is not recommended because the genetic architecture of Fusarium ear rot is based on many genes with low effects, and the power of detection would likely be reduced owing to the lower frequencies of minor alleles. Instead, searches for QTLs associated with Fusarium ear rot could be performed in other maize MAGIC populations such as the one developed by Dell'Acqua et al. (2015). Variability in Fusarium ear rot was expected in that MAGIC population because some founders differed in their resistance to Fusarium ear rot (Santiago et al. 2013). However, the usefulness of the MAGIC population developed by Dell'Acqua et al. (2015) to generate inbred lines with higher resistance to Fusarium ear rot by breeding approaches was hindered by the inclusion of inbred founders from the Reid and Lancaster heterotic groups. Therefore, the development of a Reid MAGIC population is proposed to uncover complementary genetic variability because variability among Reid materials has not been explored in the current MAGIC population, and inbreds derived from the Reid MAGIC population would presumably have good specific combining ability with inbred lines derived from our MAGIC population.

In parallel, the supporting intervals of several QTLs detected in the current study also contained SNPs that were significantly associated with resistance to Fusarium ear rot in previous association studies using inbred panels and even candidate genes for fumonisin content such as Zm00001d044175 (at chromosome 3: 221,168,477 to $221,175,604$ bp in B73_RefGen_v4, annotated as neutral/alkaline nonlysosomal ceramidase gene) and Zm00001d022400 (at chromosome 7: 176,814,267 to 176,818,536 in B73_RefGen_v4, annotated as SKP1/ASK-interacting protein 5 gene) (Chen et al. 2016; Coan et al. 2018; Ju et al. 2017; Samayoa et al. 2018; Zila et al. 2013, 2014). Therefore, the MAGIC population could be an excellent base material for performing selections for Fusarium ear rot using models that combine the effects of genetic background (that account for minor gene effects) and functional variants at validated genes (alleles for major genes identified as candidate genes using GWAS in inbred panels), because the wide variability for both types of genes could be present in this population. This breeding scheme has been proposed as the most favorable for improving traits such as Fusarium ear rot resistance that are mostly controlled by a large number of genes with small individual effects (Zila et al. 2014). However, this study was just exploratory because a subset of only approximately 340 individuals from the MAGIC population was used, and more precise studies should be performed using the complete array of 700 RILs. Simulation studies performed with another maize MAGIC population showed that increasing the number of population samples from 300 to 500 individuals almost doubles the detection power but that more than 500 samples are required for detecting QTLs with low effects (Dell' Acqua et al. 2015). Similarly, the resolutions of QTLs can be greatly improved by increasing the number of individuals because the number of observed recombination events also increases, although the use of more than 500 individuals could be unnecessary to detect QTLs that explain more than $5 \%$ of variability (Valdar et al. 2006).

In conclusion, the MAGIC population studied is a valuable tool to locate QTLs associated with resistance to Fusarium ear rot. Although it was not explored in the current work, this MAGIC population may also allow the simultaneous search for QTLs associated with resistance to fumonisin accumulation in kernels. Putative QTLs were found at bins 1.03, 1.05, 2.04, 3.01-3.02, 3.08-3.09, 4.07, 6.05, 7.02, 7.03-7.04, 7.04-7.05, 8.02, 9.03, and 10.05. The QTLs in bins 7.04-7.05, 8.02, and 9.03 were highly reliant. Present and previous results identified some crucial regions such as 210 to $220 \mathrm{Mb}$ in chromosome 3 and at 166 to $173 \mathrm{Mb}$ in chromosome 7 (B73-RefGen-v2)

\section{Distribution of SNP}

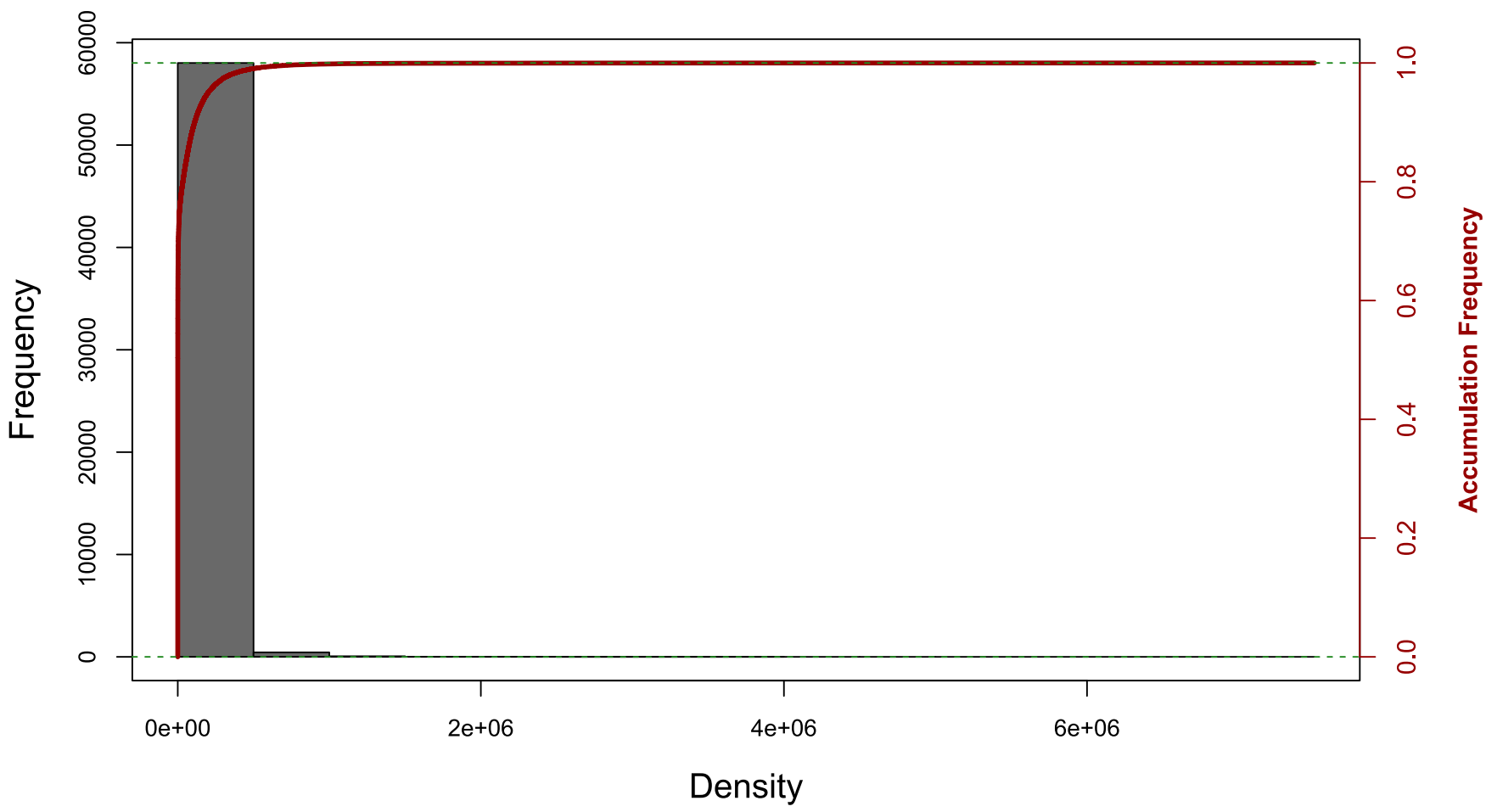

Fig. 6. Frequency and accumulative frequency of marker density. SNP = single-nucleotide polymorphism. 
which contain QTLs for Fusarium ear rot resistance and fumonisin content (Samayoa et al. 2018).

\section{Acknowledgments}

We thank B. Ordás for his contribution to MAGIC development and Ryan Sartor and Lesley Benyon for their careful language reviews.

\section{Literature Cited}

Abdel-Rahman, M. M., Bayoumi, S. R., and Barakat, M. N. 2016. Identification of molecular markers linked to Fusarium ear rot genes in maize plants Zea mays L. Biotechnol. Biotechnol. Equip. 30:692-699.

Bolduan, C., Miedaner, T., Schipprack, W., Dhillon, B. S., and Melchinger, A. E. 2009. Genetic variation for resistance to ear rots and mycotoxins contamination in early European maize inbred lines. Crop Sci. 49:2019-2028.

Bradbury, P. J., Zhang, Z., Kroon, D. E., Casstevens, T. M., Ramdoss, Y., and Buckler, E. S. 2007. TASSEL: Software for association mapping of complex traits in diverse samples. Bioinformatics 23:2633-2635.

Butrón, A., Revilla, P., Romay, M. C., Ordas, A., and Malvar, R. A. 2009. Causes of agronomic differences between synthetics developed by the random and convergent cross methods. Field Crops Res. 110:229-234.

Butrón, A., Tarrio, R., Revilla, P., Malvar, R. A., and Ordás, A. 2003. Molecular evaluation of two methods for developing maize synthetic varieties. Mol. Breed. 12:329-333.

Cao, A., Butrón, A., Ramos, A. J., Marin, S., Souto, C., and Santiago, R. 2014. Assessing white maize resistance to fumonisin contamination. Eur. J. Plant Pathol. 138:283-292

Cavanagh, C., Morell, M., Mackay, I., and Powell, W. 2008. From mutations to MAGIC: Resources for gene discovery, validation and delivery in crop plants. Curr. Opin. Plant Biol. 11:215-221.

Chen, J., Ding, J., Li, H., Li, Z., Sun, X., Li, J., Wang, R., Dai, X., Dong, H., Song, W., Chen, W., Xia, Z., and Wu, J. 2012. Detection and verification of quantitative trait loci for resistance to Fusarium ear rot in maize. Mol. Breed. 30:1649-1656.

Chen, J., Shrestha, R., Ding, J. Q., Zheng, H. J., Mu, C. H., Wu, J. Y., and Mahuku, G. 2016. Genome-wide association study and QTL mapping reveal genomic loci associated with Fusarium ear rot resistance in tropical maize germplasm. G3 Genes Genomes Genet. 6:3803-3815.

Coan, M. M. D., Senhorinho, H. J. C., Pinto, R. J. B., Scapim, C. A., Tessmann, D. J., Williams, W. P., and Warburton, M. L. 2018. Genome-wide association study of resistance to ear rot by Fusarium verticillioides in a tropical field maize and popcorn core collection. Crop Sci. 58:564-578.

Dell'Acqua, M., Gatti, D. M., Pea, G., Cattonaro, F., Coppens, F., Magris, G., Hlaing, A. L., Aung, H. H., Nelissen, H., Baute, J., Frascaroli, E., Churchill, G. A., Inze, D., Morgante, M., and Pe, M. E. 2015. Genetic properties of the MAGIC maize population: A new platform for high definition QTL mapping in Zea mays. Genome Biol. 16:23.

Ding, J.-Q., Wang, X.-M., Chander, S., Yan, J.-B., and Li, J.-S. 2008. QTL mapping of resistance to Fusarium ear rot using a RIL population in maize. Mol. Breed. 22:395-403.

Eller, M. S., Holland, J. B., and Payne, G. A. 2008. Breeding for improved resistance to fumonisin contamination in maize. Toxin Rev. 27:371-389.

Eller, M. S., Payne, G. A., and Holland, J. B. 2010. Selection for reduced Fusarium ear rot and fumonisin content in advanced backcross maize lines and their topcross hybrids. Crop Sci. 50:2249-2260.

Giomi, G. M., Kreff, E. D., Iglesias, J., Fauguel, C. M., Fernandez, M., Oviedo, M. S., and Presello, D. A. 2016. Quantitative trait loci for Fusarium and Gibberella ear rot resistance in Argentinian maize germplasm. Euphytica 211:287-294.

Gowda, M., Das, B., Makumbi, D., Babu, R., Semagn, K., Mahuku, G., Olsen, M. S., Bright, J. M., Beyene, Y., and Prasanna, B. M. 2015. Genome-wide association and genomic prediction of resistance to maize lethal necrosis disease in tropical maize germplasm. Theor. Appl. Genet. 128:1957-1968.

Holland, J. B. 2006. Estimating genotypic correlations and their standard errors using multivariate restricted maximum likelihood estimation with SAS Proc MIXED. Crop Sci. 46:642-656.

Holland, J. B. 2015. MAGIC maize: A new resource for plant genetics. Genome Biol. 16:163

Holland, J. B., Nyquist, W. E., and Cervantes-Martínez, C. T. 2003. Estimated an interpreting heritability for plant breeding: An update. Pages 9-112 in: Plant Breeding Reviews. J. Janick, ed. Vol. 22. John Wiley \& Sons, Hoboken, New Jersey.

Huang, B. E., George, A. W., Forrest, K. L., Kilian, A., Hayden, M. J., Morell, M. K., and Cavanagh, C. R. 2012. A multiparent advanced generation intercross population for genetic analysis in wheat. Plant Biotechnol. J. 10: 826-839.

Huang, B. E., Verbyla, K. L., Verbyla, A. P., Raghavan, C., Singh, V. K., Gaur, P., Leung, H., Varshney, R. K., and Cavanagh, C. R. 2015. MAGIC populations in crops: Current status and future prospects. Theor. Appl. Genet. 128:999-1017

Islam, M. S., Thyssen, G. N., Jenkins, J. N., Zeng, L. H., Delhom, C. D., McCarty, J. C., Deng, D. D., Hinchliffe, D. J., Jones, D. C., and Fang, D. D. 2016. A MAGIC population-based genome-wide association study reveals functional association of $G h R B B 1 A 07$ gene with superior fiber quality in cotton. BMC Genomics 17:903.

Jouany, J. P. 2007. Methods for preventing, decontaminating and minimizing the toxicity of mycotoxins in feeds. Anim. Feed Sci. Technol. 137:342-362.

Ju, M., Zhou, Z. J., Mu, C., Zhang, X. C., Gao, J. Y., Liang, Y. K., Chen, J. F., Wu, Y. B., Li, X. P., Wang, S. W., Wen, J. J., Yang, L. M., and Wu, J. Y. 2017. Dissecting the genetic architecture of Fusarium verticillioides seed rot resistance in maize by combining QTL mapping and genome-wide association analysis. Sci. Rep. 7: Article 46446.

Lanubile, A., Maschietto, V., Borrelli, V. M., Stagnati, L., Logrieco, A. F., and Marocco, A. 2017. Molecular basis of resistance to Fusarium ear rot in maize. Front. Plant Sci. 8:1774

Li, Z. M., Ding, J. Q., Wang, R. X., Chen, J. F., Sun, X. D., Chen, W., Song, W. B., Dong, H. F., Dai, X. D., Xia, Z. L., and Wu, J. Y. 2011. A new QTL for resistance to Fusarium ear rot in maize. J. Appl. Genet. 52:403-406.

Liu, X. L., Huang, M., Fan, B., Buckler, E. S., and Zhang, Z. W. 2016. Iterative usage of fixed and random effect models for powerful and efficient genomewide association studies. PLoS Genet. 12:e1005767.

Löffler, M., Kessel, B., Ouzunova, M., and Miedaner, T. 2011. Covariation between line and testcross performance for reduced mycotoxin concentrations in European maize after silk channel inoculation of two Fusarium species. Theor. Appl. Genet. 122:925-934.

Löffler, M., Miedaner, T., Kessel, B., and Ouzunova, M. 2010. Mycotoxin accumulation and corresponding ear rot rating in three maturity groups of European maize inoculated by two Fusarium species. Euphytica 174: 153-164.

Maschietto, V., Colombi, C., Pirona, R., Pea, G., Strozzi, F., Marocco, A., Rossini, L., and Lanubile, A. 2017. QTL mapping and candidate genes for resistance to Fusarium ear rot and fumonisin contamination in maize. BMC Plant Biol. 17: 20

Maschietto, V., Marocco, A., Malachova, A., and Lanubile, A. 2015. Resistance to Fusarium verticillioides and fumonisin accumulation in maize inbred lines involves an earlier and enhanced expression of lipoxygenase (LOX) genes. J. Plant Physiol. 188:9-18.

Meng, L. J., Guo, L. B., Ponce, K., Zhao, X. Q., and Ye, G. Y. 2016a. Characterization of three Indica rice multiparent advanced generation intercross (MAGIC) populations for quantitative trait loci identification. Plant Genome 9:1-14.

Meng, L. J., Zhao, X. Q., Ponce, K., Ye, G. Y., and Leung, H. 2016b. QTL mapping for agronomic traits using multi-parent advanced generation intercross (MAGIC) populations derived from diverse elite Indica rice lines. Field Crops Res. 189:19-42.

Pascual, L., Albert, E., Sauvage, C., Duangit, J., Bouchet, J. P., Bitton, F., Desplat N., Brunel, D., Le Paslier, M. C., Ranc, N., Bruguier, L., Chauchard, B. Verschave, P., and Causse, M. 2016. Dissecting quantitative trait variation in the resequencing era: Complementarity of bi-parental, multi-parental and association panels. Plant Sci. 242:120-130.

Pascual, L., Desplat, N., Huang, B. E., Desgroux, A., Bruguier, L., Bouchet, J. P., Le Q. H., Chauchard, B., Verschave, P., and Causse, M. 2015. Potential of a tomato MAGIC population to decipher the genetic control of quantitative traits and detect causal variants in the resequencing era. Plant Biotechnol. J. 13:565-577.

Pérez-Brito, D., Jeffers, D., González-de-León, D., Khairallah, M., Cortés-Cruz, M., Velázquez-Cardelas, G., Azpíroz-Rivero, S., and Srinivasan, G. 2001. QTL Mapping of Fusarium moniliforme ear rot resistance in highland maize, Mexico. Agrociencia 35:181-196.

Presello, D. A., Pereyra, A. O., Iglesias, J., Fauguel, C. M., Sampietro, D. A., and Eyherabide, G. H. 2011. Responses to selection of S-5 inbreds for broad-based resistance to ear rots and grain mycotoxin contamination caused by Fusarium spp. in maize. Euphytica 178:23-29.

Reid, L. M., Hamilton, R. E., and Mather, D. E. 1996. Screening Maize for Resistance to Gibberella Ear Rot. Tech. Bull. 62. Agriculture and Agri-Food Canada, Ottawa, ON, Canada.

Reid, L. M., and Zhu, X. 2005. Screening Corn for Resistance to Common Diseases in Canada. Tech. Bull. Agriculture and Agri-Food Canada, Ottawa, ON, Canada

Robertson, L. A., Kleinschmidt, C. E., White, D. G., Payne, G. A., Maragos, C. M., and Holland, J. B. 2006. Heritabilities and correlations of Fusarium ear ro resistance and fumonisin contamination resistance in two maize populations. Crop Sci. 46:353-361.

Robertson-Hoyt, L. A., Jines, M. P., Balint-Kurti, P. J., Kleinschmidt, C. E., White, D. G., Payne, G. A., Maragos, C. M., Molnar, T. L., and Holland, J. B. 2006. QTL mapping for Fusarium ear rot and fumonisin contamination resistance in two maize populations. Crop Sci. 46:1734-1743.

Samayoa, L. F., Santiago, R., Malvar, R. A., Cao, A., and Butrón, A. 2018. A genome-wide association analysis study (GWAS) for fumonisin content in maize kernel. Page 88 in: EFS14, 14th Eur. Fusarium Semin. Tulln, Austria.

Sannemann, W., Huang, B. E., Mathew, B., and Leon, J. 2015. Multi-parent advanced generation inter-cross in barley: High-resolution quantitative trait locus mapping for flowering time as a proof of concept. Mol. Breed. $35: 86$.

Santiago, R., Cao, A., and Butrón, A. 2015. Genetic factors involved in fumonisin accumulation in maize kernels and their implications in maize agronomic management and breeding. Toxins (Basel) 7:3267-3296. 
Santiago, R., Cao, A., Malvar, R. A., Reid, L. M., and Butrón, A. 2013. Assessment of corn resistance to fumonisin accumulation in a broad collection of inbred lines. Field Crops Res. 149:193-202.

SAS Institute. 2008. SAS/STAT 9.2 User's Guide. SAS Institute Inc., Cary, NC.

Valdar, W., Flint, J., and Mott, R. 2006. Simulating the collaborative cross: Power of quantitative trait loci detection and mapping resolution in large sets of recombinant inbred strains of mice. Genetics 172:1783-1797.
Verbyla, A. P., George, A. W., Cavanagh, C. R., and Verbyla, K. L. 2014. Wholegenome QTL analysis for MAGIC. Theor. Appl. Genet. 127:1753-1770.

Zila, C. T., Fernando Samayoa, L., Santiago, R., Butrón, A., and Holland, J. B. 2013. A genome-wide association study reveals genes associated with Fusarium ear rot resistance in a maize core diversity panel. G3 Genes Genomes Genet. 3:2095-2104.

Zila, C. T., Ogut, F., Romay, M. C., Gardner, C. A., Buckler, E. S., and Holland, J. B. 2014. Genome-wide association study of Fusarium ear rot disease in the U.S.A. maize inbred line collection. BMC Plant Biol. 14:372. 